\title{
Transcriptional Profiling of Soft-rot Resistant Transgenic Chinese Cabbage (Brassica rapa L.) Constitutively Overexpressing a Human Cathelicidin Antimicrobial Peptide (hCAP18/LL-37)
}

\author{
Yu-Jin Jung ${ }^{1,2} *$ \\ ${ }^{1}$ Department of Horticulture, Hankyong National University, Anseong 456-749, Korea \\ ${ }^{2}$ Institute of Genetic Engineering, Hankyong National University, Ansung 456-749, Korea
}

\begin{abstract}
Soft rot disease caused by Pectobacterium carotovorum subsp. carotovorum (Pcc), is one of the most devastating diseases affecting the cruciferous plants industry worldwide. In our previous study, the soft rot-resistant transgenic Chinese cabbage (Brassica rapa L.) plants were produced via constitutively overexpressing a human cathelicidin antimicrobial peptide (hCAP18/LL-37). To unravel the molecular mechanisms underlying Pcc resistance of the transgenic plants, this study compares the global transcriptional profile of untransformed line (WT) and the transgenic lines (TG23, TG34) through hybridization with KBGP-24K, Chinese cabbage GeneChip. In total, 1,415 differentially expressed genes (DEGs) were identified, 910 of which were up-regulated, while 505 were down-regulated. The DEGs were classified into 31 categories after Gene ontology (GO) annotation, in which 68 genes are in response to stimulus and are involved in immune system process, 12 genes are related to cell wall, and 13 genes belong to transcription factors. These genes and those related to toxin and terpenoid metabolism, glutathione metabolism, biosynthesis of phenylpropanoids, and plant hormones were hypothesized to play major roles in the soft rot resistance of transgenic lines (TG23, TG34). Semiquantitative RT-PCR analysis showed that the transcript levels of several candidate genes in TG23 and TG34 were significantly higher than in WT both before and after Pcc inoculation, indicating their potential association with soft rot disease.
\end{abstract}

Keywords Antimicrobial peptide (hCAP18/LL-37), Chinese cabbage, Microarray analysis, Soft rot resistance, Transcriptional profiling, Transgenic plant

\section{INTRODUCTION}

Bacterial and fungal plant pathogens severely affect crop productivity. For example, Xanthomonas campestris pv. campestris and Pectobacterium carotovorum subsp. carotovorum, which cause black rot and soft rot, respectively, are present worldwide and severely damage plants and reduce their yields, especially in cruciferous plants (Boman 2003). Therefore, the development of cruciferous plants that are resistant to black and soft rot diseases has been a major goal of researchers for several decades. Strategies based on transgenic approaches to enhance plant disease resistance involve the use of genes associated with plant defense pathways (Makandar et al. 2006; Zhang et al. 2007) and genes encoding plant or fungal hydrolytic enzymes (Bieri et al. 2003), defense-related transcription factors (Chen and Chen 2002; Sohn et al. 2006) and antimicrobial peptides (Alan et al. 2004). A large number of antimicrobial peptides from different organisms have been characterized (Simmaco et al. 1998). In our previous study, we produced transgenic Chinese cabbage plants with less susceptibility to soft rot via constitutively overexpressing a human cathelicidin antimicrobial peptide (hCAP18/LL-37) (Jung et al. 2012). The human cathelicidin antimicrobial protein hCAP18 is the only member of the mammalian cathelicidin family of proteins that is present in

Received Mar 18, 2013; Revised Mar 22, 2013; Accepted Mar 22, 2013; Published March 31, 2013

*Corresponding author Yu-Jin Jung, yuyu1216@hknu.ac.kr, Tel: +82-31-678-4642, Fax: +82-31-670-5109 
humans (Gudmundsson et al. 1996). The holoprotein consists of a conserved prodomain, a cathelin domain, and the non-conserved C-terminal peptide LL-37, which is enzymatically cleaved after secretion (Sorensen et al. 2001; Yamasaki et al. 2006). Its precursor molecule, an $18 \mathrm{kDa}$ human cationic antimicrobial protein (hCAP-18), is secreted by activated neutrophil granulocytes. After release, the helical C-terminal end of this precursor comprising 37 amino acids is cleaved off, thereby forming the functional antimicrobial peptide LL-37 (Sorensen et al. 2001). Since LL-37 is the only human antimicrobial peptide that is active at physiological or elevated salt concentration conditions, there is a significant interest in using this peptide for pharmaceutical applications (De Smet and Contreras 2005; Reddy et al. 2004; Travis et al. 2000). In our previous study, the soft rot-resistant transgenic Chinese cabbage (Brassica rapa L.) plants were produced via constitutively overexpressing a human cathelicidin antimicrobial peptide (hCAP18/LL-37). To gain new insights into the molecular mechanisms of enhanced disease tolerance, genome-wide transcriptome analysis was conducted using the KBGP-24K, GeneChip microarray technology. The transcriptional profiling described here may contribute to explain molecular mechanism of polyamine in regulating plant pathogen response.

\section{MATERIALS AND METHODS}

\section{Microarray hybridization and data analysis}

The leaves were collected from untransformed (WT) and transgenic lines (TG23, TG34) for hybridization with the GeneChip (http://www.brassica-rapa.org/BrMED/microarray_ overview.jsp). In brief, two g leaves of WT, TG23 and TG34 were sampled from uniform new flushes (about 20 days after sprout) and then immediately immersed in liquid nitrogen and stored at $-80^{\circ} \mathrm{C}$. All the other processes including the total RNA extraction (20 $\mu \mathrm{g}$ at least), cDNA synthesis, cDNA fragmentation, hybridization, washing and staining, and scanning were performed as reported by Lee et al. (2008). To satisfy biological reproducibility requirements, the experiment was carried out using three independent biological replicates for both WT, TG23, TG34 (means among WT, TG23 and TG34 were hybridized with microarray for three times). The probe array was scanned with the GenePix scanner 4000B (Axon), and the images were analyzed with the NimbleScan (NimbleGen). The data were normalized and processed with cubic spline normalization using quantiles to adjust signal variations between chips and with Rubust Multi-Chip Analysis (RMA) using a median polish algorithm implemented in NimbleScan software (NimbleGen) (Irizarry et al. 2003; Workman et al. 2002). Analysis of variance (ANOVA) was used to compare the statistical expression difference between TG23 and WT. Probe sets with a $P$ value $\leq 0.05$ and 2-fold change were considered as differentially expressed genes (DEGs) between the two groups at a statistically significant level.

\section{Microarray annotation and functional analysis}

To assign putative functions of DEGs, Gene ontology (GO) term, Enzyme Commission (EC), and Kyoto Encyclopedia of Genes and Genomes (KEGG) annotation were performed using the Blast2GO (Conesa et al. 2005) software. Blast2GO assigns $\mathrm{GO}$ annotation through three steps, blasting, mapping, and annotation. GO terms for each of the three main categories (biological process, molecular function, and cellular component) was obtained by using the combined graphs function of the software with default parameters. The KEGG analysis were performed by using the KEGG annotating function of Blast2GO software, and the annotated KEGG pathways were further manually classified according to the published KEGG pathway lists (http://www.genome.jp/kegg/pathway.html).

\section{Semiquantitative RT-PCR analysis}

Semiquantitative RT-PCR was employed to validate the microarray results using the RNA samples for the hybridization experiments. Each RNA sample was pretreated with PCR amplification-grade RNase-free DNase I (Promega, Korea) at $37^{\circ} \mathrm{C}$ to exclude DNA contamination. cDNA synthesis was done using Reverse Trascriptase kit (Toyobo, Japan) following the manufacturer's instructions. Specific primers of candidate genes were designed by Primer Premier 5.0 software (PRIMER Biosoft International, Palo Alto, CA) based on the Chinese cabbage consensus sequences downloaded from Brassica rapa Genome Project website 
(http://brassicadb.org/brad/). Each PCR reaction was composed of $50 \mathrm{ng}$ cDNA, $2.0 \mu \mathrm{L} 10 \times$ reaction buffer, 1.0 $\mathrm{mM} \mathrm{MgCl} 2,0.2 \mathrm{mM}$ dNTP, $1.0 \mathrm{U}$ of DNA polymerase (Taq, Takara) and $0.4 \mu \mathrm{M}$ of each primer in a total volume of $20 \mu \mathrm{L}$. PCR amplifications were performed at $94^{\circ} \mathrm{C}$ for $5 \mathrm{~min}$, followed by $28-32$ cycles of $94^{\circ} \mathrm{C}$ for $40 \mathrm{~s}, 52^{\circ} \mathrm{C}$ for $40 \mathrm{~s}, 72^{\circ} \mathrm{C}$ for $40 \mathrm{~s}$ and $5 \mathrm{~min}$ extension at $72^{\circ} \mathrm{C}$. A BrActin gene (Table 1) was used as an internal positive control. Band intensity was quantified by Quantity One analysis software (Bio-Rad Laboratories), and the fold change was calculated by the signal intensity of TG23, TG34-specific product divided by the signal intensity of WT-specific product. In another experiment, semiquantitative RT-PCR was performed to evaluate the expression patterns of several genes before or after Pectobacterium carotovorum subsp. carotovorum $(P c c)$ inoculation. For this purpose, the leaves sampled from uniform new flushes (about 20 days after sprout) of WT, TG23 and Tg34 were divided into two groups, respectively. One group of leaves without $P c c$ inoculation (uninoculated leaves) were immediately immersed in liquid nitrogen and stored at $-80^{\circ} \mathrm{C}$. And another group of leaves were subjected to a pinprick inoculation with $P c c$ bacterial suspension as described by Jung et al. (2008). 48 hours after inoculation (hpi), the whole leaves of WT, TG23 and TG34 were collected and stored at $-80^{\circ} \mathrm{C}$. The total RNA was isolated from uninoculated ( $0 \mathrm{hpi})$ and inoculated leaves ( $24 \mathrm{hpi}$ and $48 \mathrm{hpi}$ ) according to Jung et al. (2008). The other processes including RNA pretreatment, cDNA synthesis, PCR amplification, and quantification of band intensity were the same as mentioned above.

\section{RESULTS}

\section{Screening of the differentially expressed genes and verifying the microarray data}

In our previous study, we produced transgenic Chinese cabbage plants with less susceptibility to soft rot via

Table 1. Significantly twenty up-regulated genes among genes in the LL-37 transgenic plants and wild type using KBGP-24K oligo chip.

\begin{tabular}{ccll}
\hline \hline $\begin{array}{c}\text { Clone } \\
\text { number }\end{array}$ & $\begin{array}{c}\text { Fold over } \\
\text { expression }\end{array}$ & Gene ID & \multicolumn{1}{c}{ Description } \\
\hline 28869 & 48.87 & EAK95476 & Potential purine-cytosine permease \\
26645 & 31.37 & AT1G62940 & Similar to ataxia/oculomotor apraxia protein 2 \\
22041 & 29.07 & AAL91065 & NADH dehydrogenase subunit 6 \\
00544 & 21.29 & AT1G12520 & Cu/Zn superoxide dismutase copper chaperone like protein \\
00089 & 20.49 & AT1G20693 & Chromatin-associated proteins \\
24612 & 18.98 & AAG51989 & Glyoxalase II, putative \\
02138 & 18.76 & AT1G20760 & Calcium-binding EF hand family protein \\
01218 & 17.32 & AT4G25780 & PR-1 protein from Medicago truncatula \\
15181 & 17.29 & AT1G22080 & Unknown protein \\
19064 & 13.74 & AT4G22880 & Anthocyanidin synthase \\
19451 & 13.20 & AT1G64160 & Disease resistance-responsive family protein \\
11475 & 12.89 & AT5G44420 & Plant defensin protein family \\
00303 & 12.69 & AT4G25100 & Superoxide dismutase \\
01138 & 6.72 & AT1G27310 & Similar to nuclear transport factor 2 \\
07649 & 4.68 & AT1G06830 & Glutaredoxin, putative \\
25621 & 4.32 & AT2G21100 & Disease resistance-responsive protein \\
04541 & 4.25 & AT5G38195 & Protease inhibitor contains lipid transfer protein (LTP) \\
19333 & 3.79 & AT4G19880 & Glutathione S-transferase-related \\
29028 & 3.71 & AT1G28520 & Putative myosin heavy chain \\
15849 & 3.39 & AT5G57350 & ATPase \\
\hline
\end{tabular}




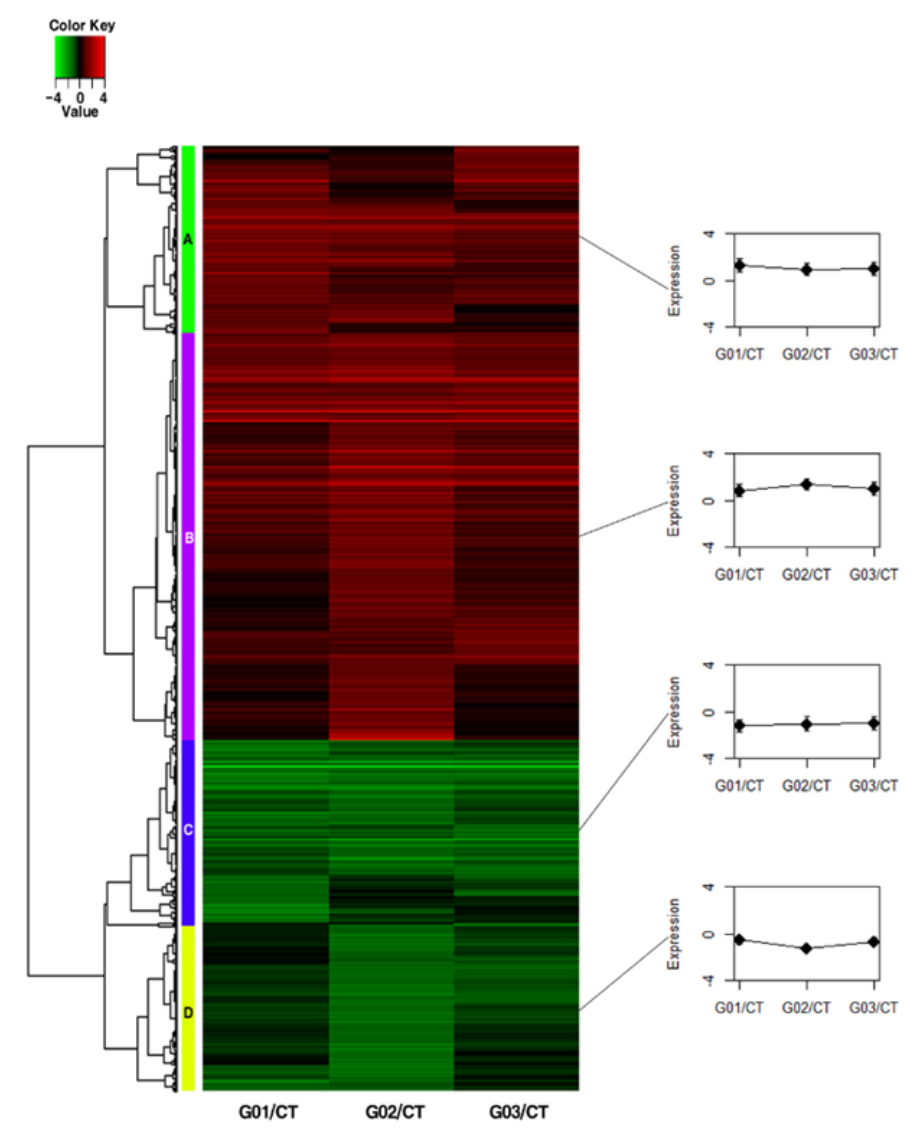

Fig. 1. Hierarchical clustering among the genes highly expressed antimicrobial peptide LL-37 transgenic and wild type plants in Brassica rapa using KBGP-24K chip. Four representative patterns (A D) displayed similar expression patterns, respectively. Expression values higher and lower than those of the control are shown in red and green, respectively.

constitutively overexpressing a human cathelicidin antimicrobial peptide (hCAP18/LL-37) (Jung et al. 2012). To reveal the molecular mechanisms underlying soft rot resistance in transgenic lines, the global transcriptional profile of transgenic lines (TG23, TG34) and WT were compared by Chinese cabbage genome GeneChip analysis. After statistical analysis, 1,415 genes with signal ratio fold change larger than 2 or smaller than $0.5(P$ value $\leq 0.05)$ between the transgenic lines and WT were identified as differentially expressed genes (DEGs). Among these genes, 910 were up-regulated and 505 were down-regulated (Fig. 1). In order to verify the reliability of the microarray data, 20 up-regulated and 20 down-regulated genes were randomly selected to analyze their expression levels in transgenic lines and WT via semiquantitative RT-PCR using genespecific primers. The up-regulated genes ID are potential purine-cytosine permease (EAK95476), ataxia/oculomotor apraxia protein (AT1G62940), NADH dehydrogenase subunit (AAL91065), $\mathrm{Cu} / \mathrm{Zn}$ superoxide dismutase copper chaperone like protein (AT1G12520), calcium-binding EF hand family protein (AT1G20760), PR-1 protein (AT4G25780), disease resistance-responsive family protein (AT1G64160) and protease inhibitor contains lipid transfer protein (LTP) (AT5G38195). However, the genes ID of down-regulated to low levels, and 20 out of 218 genes with $\log _{2}$ ratios lower than 0.5 are putative $\mathrm{CCCH}$-type zinc finger protein (AT4G29190), novel cap-binding protein nCBP (AT5G18110), WRKY family transcription factor (AT4G18170), polygalacturonase inhibiting protein (AT5G06860), lectin like protein (AT4G19840), trichohyalin (CAH70024) and galactinol synthase-like protein (AT2G02930) (Table 2). 
- Plant Breed. Biotech. 2013 (March) 1(1):80 90

Table 2. Significantly twenty down-regulated genes among genes in the LL-37 transgenic plants and wild type using KBGP-24K oligo chip.

\begin{tabular}{cccl}
\hline \hline $\begin{array}{c}\text { Clone } \\
\text { number }\end{array}$ & $\begin{array}{c}\text { Fold down } \\
\text { expression }\end{array}$ & Gene ID & \multicolumn{1}{c}{ Description } \\
\hline 19596 & 0.39 & AT4G29190 & Putative CCCH-type zinc finger protein \\
03882 & 0.38 & AT4G38960 & RNA-directed DNA polymerase-like protein \\
14945 & 0.37 & AT5G18110 & Novel cap-binding protein nCBP \\
07928 & 0.37 & AT4G18170 & WRKY family transcription factor, similar to DNA-binding protein 2 \\
16572 & 0.36 & AT5G62360 & Unknown protein \\
21724 & 0.36 & AT5G06860 & Polygalacturonase inhibiting protein 1 \\
02287 & 0.35 & AT5G05340 & PERP7_BRARA Peroxidase P7 (TP7) \\
02119 & 0.35 & AT3G55770 & Putative transcription factor L2 \\
00753 & 0.34 & AT5G06730 & Putative peroxidase \\
19993 & 0.33 & AT4G19840 & Lectin like protein \\
15333 & 0.33 & AT3G53140 & Caffeic acid O-methyltransferase-like protein \\
14083 & 0.32 & AT3G18490 & Putative chloroplast nucleoid DNA-binding protein \\
23389 & 0.32 & CAH70024 & Trichohyalin \\
04373 & 0.28 & AT4G02980 & Susceptible endoplasmic reticulum auxin-binding protein 2 \\
14555 & 0.19 & AT2G02930 & Glutathione S-transferase 6 \\
02692 & 0.17 & AT5G02020 & Putative protein \\
06607 & 0.15 & AT1G56600 & Galactinol synthase-like protein \\
24949 & 0.14 & XP_416059 & Similar to SF21 protein \\
00671 & 0.09 & AT2G30490 & Cinnamate-4-hydroxylase \\
02751 & 0.06 & AT1G80130 & Unknown protein \\
\hline
\end{tabular}

\section{Functional annotation and classification of the differentially expressed genes}

To further analyze the microarray data, the identified DEGs, including significantly up-regulated and downregulated genes, were functionally annotated and classified using Blast2GO software. The functional categorization was performed according to biological process, molecular function, and cellular component using Blast2GO software. As shown in Fig. 2, the biological processes of these DEGs included mainly 16 categories such as cellular process, metabolic process, response to stimulus, localization and biological regulation, and among which the genes in response to stimulus and immune system process are of interest because they may participate in soft rot disease resistance directly. In addition, it is intriguing to find that most of these categories contained larger number of the up-regulated genes than the down-regulated genes, such as cellular process, metabolic process, response to stimulus, and so forth. In the immune system process, only upregulated genes were assembled to this group. Molecular functions were primarily related to catalytic activity, transporter activity, electron carrier activity, transcription regulator activity, and others (Fig. 2). Cellular component included cell, organelle, macromolecular complex, extracellular region, membrane-enclosed lumen, and envelope. Similar to the biological process category, in molecular function and cellular component the number of up-regulated genes is larger than that of down-regulated genes (Fig. 2).

\section{Validation of microarray data by real-time PCR}

In order to validate our microarray results we performed quantitative real time PCR (qRT-PCR) to determine the expression levels of twenty genes selected from the list of up and down regulated genes (Table 1 and 2). A complete 


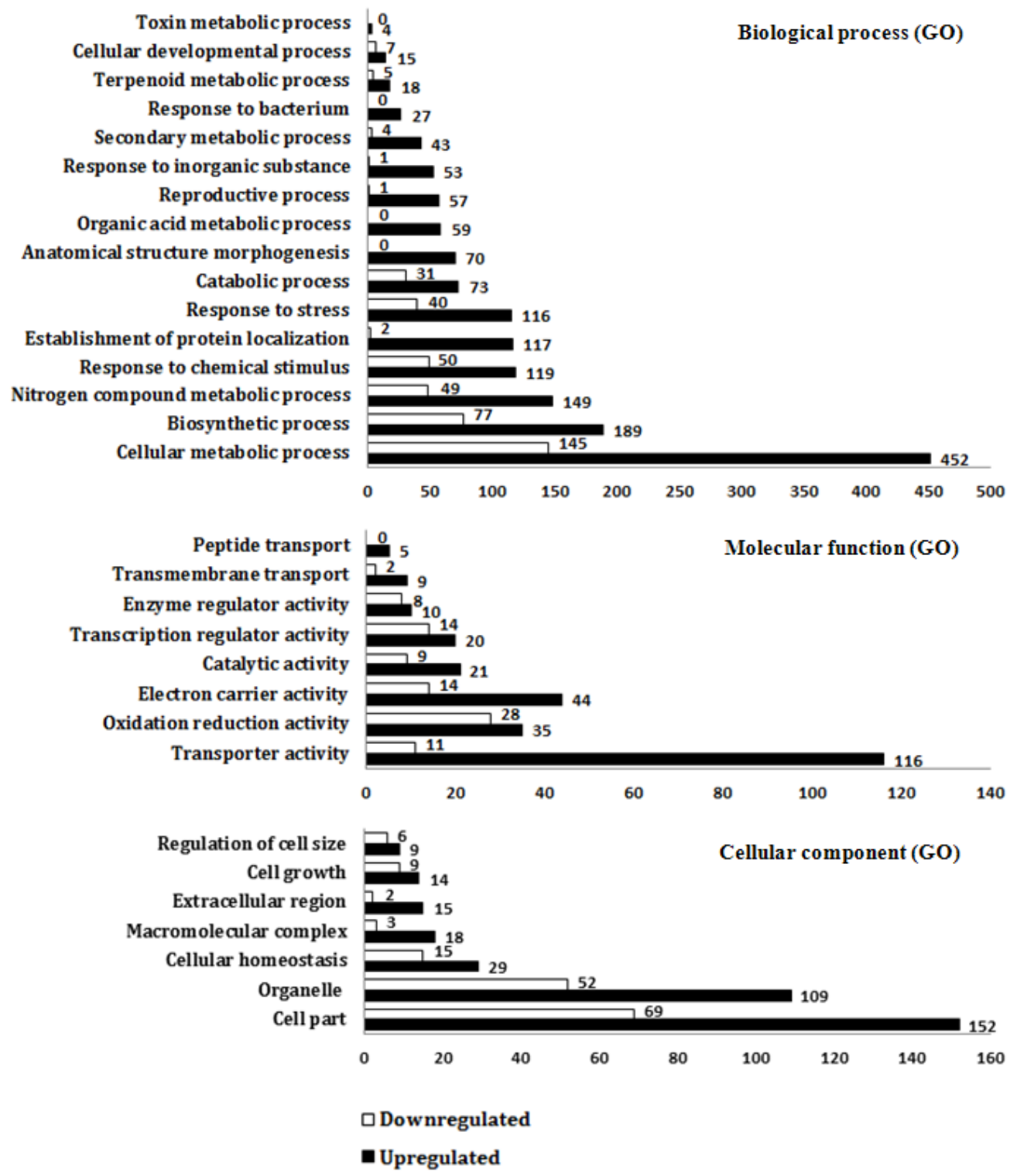

Fig. 2. Functional categorization of upregulated and downregulated differentially expressed genes. 910 significantly upregulated and 505 significantly downregulated DEGs were categorized to biological process, molecular function, and cellular component based on GO annotation, and the represented number of each column was marked in the figure.

correlation of expression results in qRT-PCR and microarray was demonstrated in this study (Fig. 3). qRT-PCR determination of LL-37 transgenic and WT plants mRNA levels showed 10-fold increase in potential purine-cytosine permease, ataxia/oculomotor apraxia protein, $\mathrm{Cu} / \mathrm{Zn}$ superoxide dismutase copper chaperone like protein, calcium-binding EF hand family protein, PR-1 protein, unknown protein, disease resistance-responsive family protein, plant defensin protein family and protease inhibitor contains lipid transfer protein (LTP). In contrast, WRKY family transcription factor, polygalacturonase inhibiting protein, putative peroxidase, lectin like protein, trichohyalin, glutathione Stransferase, unknown protein and galactinol synthase-like protein exhibited a 10-fold decrease. These results demonstrate which and to what extent genes were actively expressed upon intrusion of plant pathogen. 

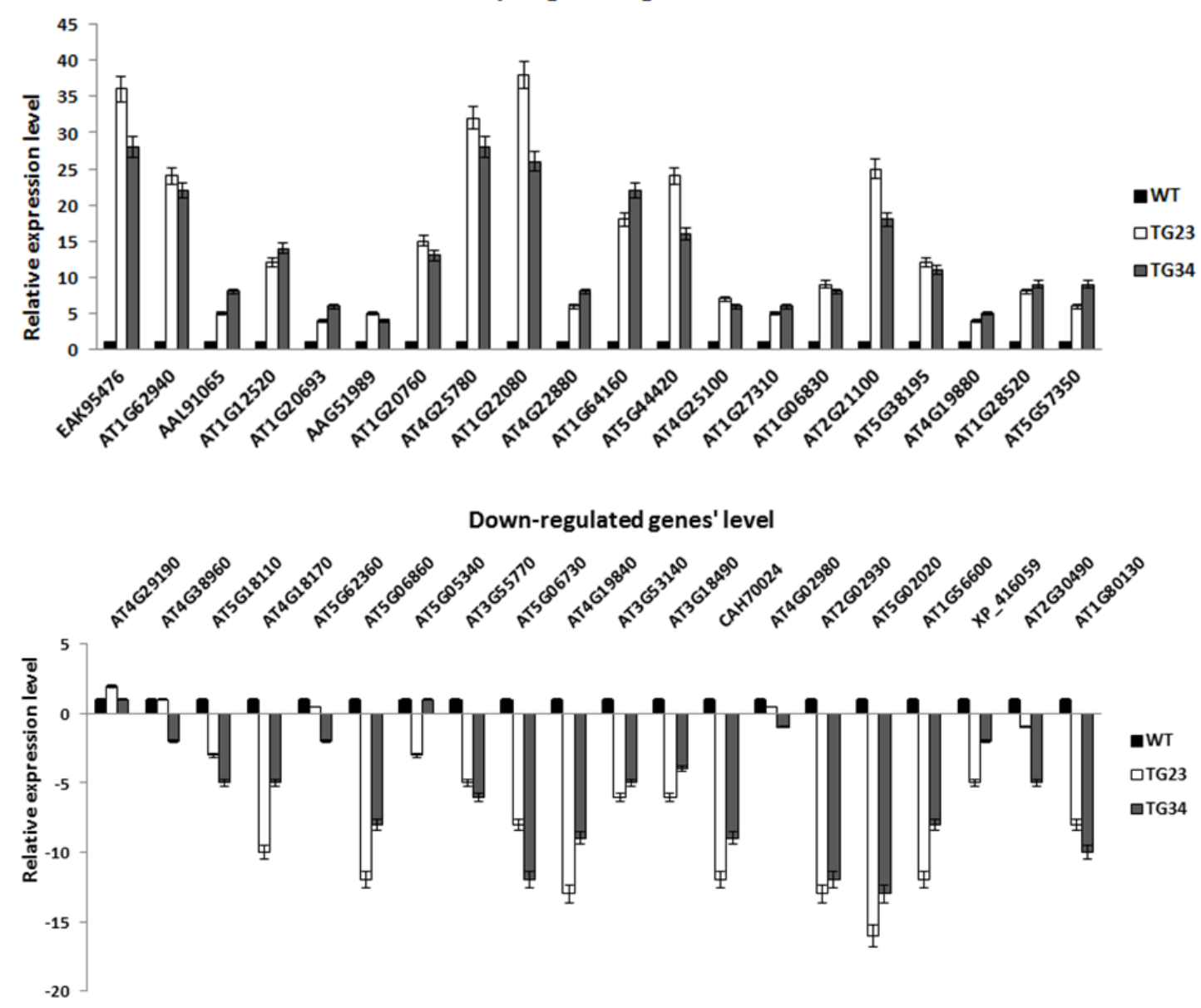

Fig. 3. Validation of microarray data. Each of twenty genes of up- and down-regulated genes showing differential expression in our microarray experiments was selected and their relative expression determined using qRT-PCR.

\section{The expression of candidate genes before and after inoculation}

The homozygous lines harboring the human LL-37 gene were evaluated for resistance to soft rot pathogen. Transgenic and control plants were inoculated with conidia, and the sizes of the disease lesions were determined. In order to test the resistance of the transgenic plants, we cultivated the cabbage soft rot pathogen $P$. carotovorum subsp. carotovorum on LB medium for 1-2 days. The whole leaf blade and leaf vein were infiltrated with $10^{8} \mathrm{CFU} / \mathrm{mL}$ inoculum; subsequently, the pathogenesis at the leaf was examined at 24,48 , and 72 $\mathrm{h}$ after inoculation. The control cabbage plants displayed the symptoms $24 \mathrm{~h}$ after inoculation where leaves became softer with visible lesions. After $48 \mathrm{~h}$, the symptom spread all over the leaves of the control plant which eventually died after $72 \mathrm{~h}$ due to roting and softening of the tissues. The wild type plants showed considerably 2 -fold higher in disease severity compared with transgenic plants (Fig. 4A and B). After $48 \mathrm{~h}$ of inoculation, the highly expressed genes identified in transgenic plants were chaperone like protein gene, PR-1 protein gene, unknown protein gene, disease resistance-responsive protein gene, plant defensin protein gene, superoxide dismutase gene, protease inhibitor contains lipid transfer protein (LTP) gene and glutathione S-transferase-related genes (Fig. 4C). Our data suggested that the expression of these candidate genes were constitutively up-regulated in the transgenic line and can be further induced by the $P c c$ inoculation, providing important information and evidence for its potential role in soft rot disease resistance. 
A
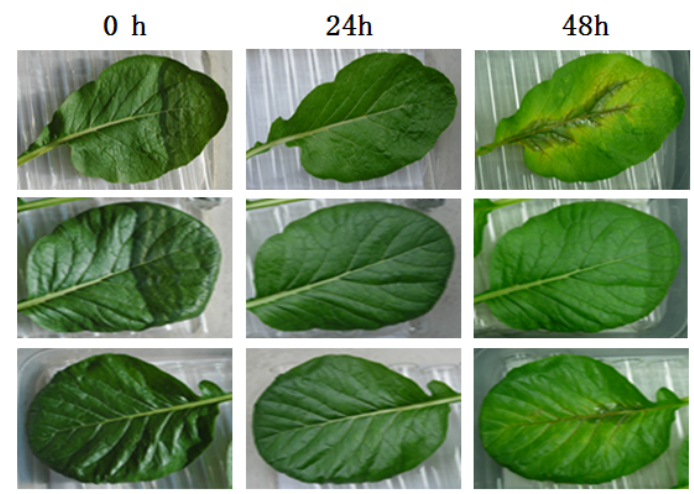

B

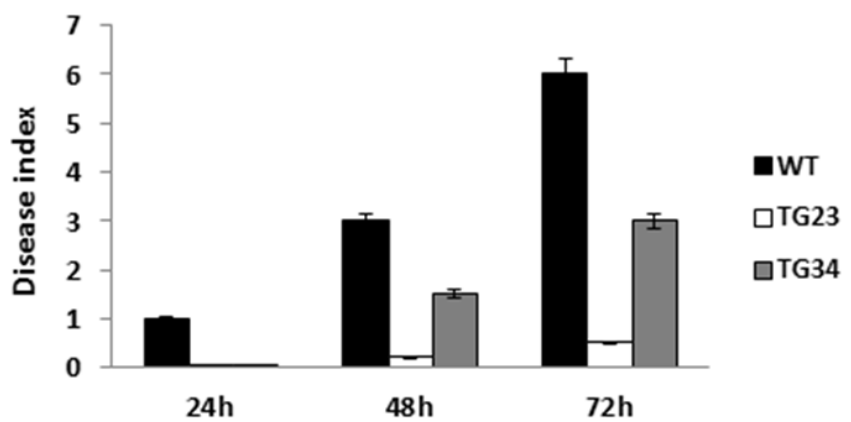

C

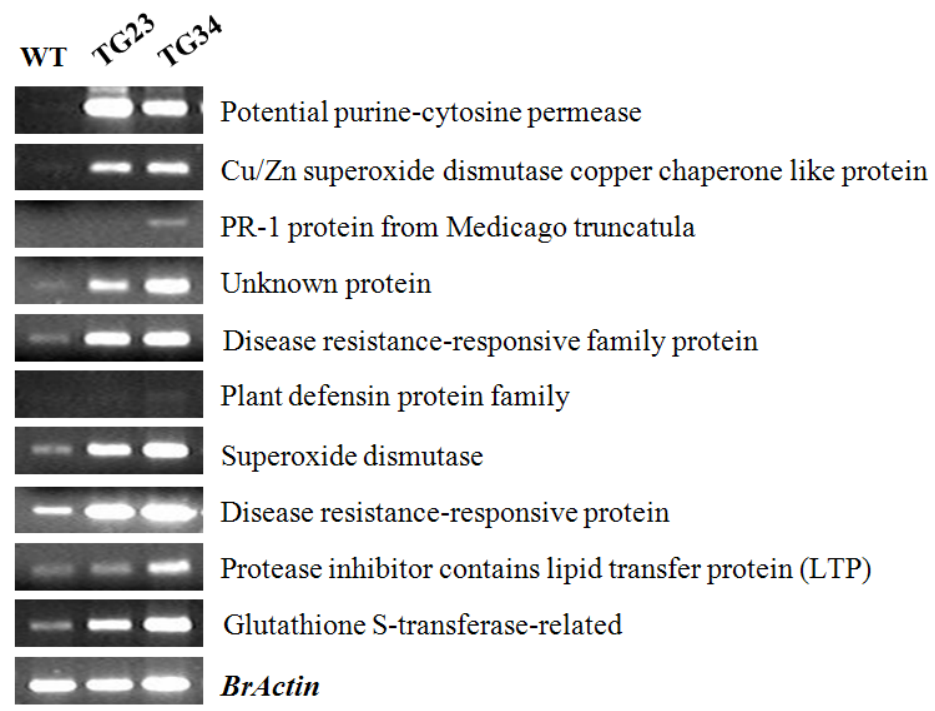

Fig. 4. Disease response and RT-PCR analysis in transgenic lines (TG23, TG34) and wild type. (A), (B) Enhancing bacterial disease resistance in transgenic plants to 3 days leaf after infection. (B) Semi-quntitative RT-PCR analysis of expression levels of candidate genes identified by microarray analysis in LL-37 transgenic and wild type control inoculated with $10^{8} \mathrm{CFU} / \mathrm{ml}$ of $P$. carotovorum ssp. carotovorum after 48 hours.

\section{DISCUSSION}

cDNA arrays were used to monitor transcript levels of LL-37 in transgenic plant, and as a gene discovery tool to identify genes whose expression is regulated during the plant-pathogen interaction. Bacterial plant pathogens severely affect crop productivity. A large number of antimicrobial peptides from different organisms have been characterized (Simmaco et al. 1998). The human cathelicidin antimicrobial protein hCAP18, which includes the C-terminal peptide 
LL-37, is a multifunctional protein (Jung et al. 2011; Kim and Martin 2004; Kunkel 2002). Strategies based on transgenic approaches to enhance plant disease resistance involve the use of genes associated with plant defense pathways (Makandar et al. 2006, Zhang et al. 2007) and genes encoding plant or fungal hydrolytic enzymes (Bieri et al. 2003), defense-related transcription factors (Chen and Chen 2002, Sohn et al. 2006) and antimicrobial peptides (Alan et al. 2004). When induced defense responses are rapidly and coordinately triggered during a given plant ${ }^{-}$ pathogen interaction, plants become broadly resistant to diseases. These defense responses include the strengthening of mechanical barriers, oxidative burst, and production of antimicrobial compounds (Hammond-Kosack and Parker 2003; Park 2005). Some research has been performed to bolster plant defenses against bacteria and fungi by genetically engineering plants to express antimicrobial peptides (Lee et al. 2008; Prasad et al. 2008). Previously, we developed homozygous Chinese cabbage lines stably expressing LL-37, which did not cause adverse effects on the plant phenotypes (Jung et al. 2012). LL-37 peptide was properly targeted to the extracellular space even with a foreign plant signal peptide. Furthermore, the mammalian peptide was not subjected to a processing step in the foreign plant cell environment that rendered it inactive. Until now, studies involving the enhancement of resistance to various bacterial, fungal, and oomycete pathogens by the expression of antimicrobial peptides have been reported for rice, tobacco, poinsettia, banana, and more host species (Chakrabarti et al. 2003; Liang et al. 2002; Smith et al. 1998). However, progress on identifying the defense mechanisms in Chinese cabbage (B. rapa), an important vegetable crop in Asia, has been very slow. Here, we demonstrate that related gene expression by the human LL-37 peptide has antimicrobial activity through microarray analysis. Also, our pathogenicity assays suggest that the expression of LL-37 confers moderate level of resistance against $P$. carotovorum subsp. carotovorum at the inoculum concentration of $10^{8} \mathrm{CFU} / \mathrm{mL}$ (Fig. 4A and B). Results of microarray analysis revealed the up-regulation of defensin protein such as PR-1 protein, plant defensin protein, disease resistance-responsive protein and LTP protein in the transgenic LL-37 plant over the WT (Table 1 and Fig. 4C). Defense-response genes that encode
PR proteins and enzymes of phytoalexin biosynthesis, show transient increases in expression during the early stages of the symbiosis and then the transcript levels subsequently decline (Harrison and Dixon 1993; GianinazziPearson et al. 1996). On the other hand, down-regulation genes revealed inhibiting protein or transcription factor such as trichohyalin, polygalacturonase inhibiting protein, lectin like protein, $\mathrm{CCCH}$-type zinc finger protein and WRKY transcription factor. Trichohyalin-like proteins regulate cell-cell interactions and communication, and provide key structural, positional and environmental signals during growth and development (Kim et al. 2003; Markus et al. 2006). Also, WRKY transcription factors (Rizhsky et al. 2002; Kalde et al. 2003) and zinc finger protein (Blackshear PJ 2002; Wang L et al. 2008) have also been implicated in plant defense to environmental stresses. The expression of the human LL-37 peptide is expected to confer durable resistance to a wide variety of pathogens infecting Chinese cabbage plants. Thus, other antimicrobial genes such as the bromelain gene (Jung et al. 2008) among others could be stacked with the human LL-37 gene by crossing different transgenic lines and this may be able to strongly and durably inhibit the growth of pathogens. The possible contribution of up-regulation of genes encoding proteins associated with the enhanced stress tolerance in our transgenic Chinese cabbage however requires further investigation.

\section{REFERENCES}

Alan AR, Blowers A and Earle ED. 2004. Expression of a magainin-type antimicrobial peptide gene (MSI-99) in tomato enhances resistance to bacterial speck disease. Plant Cell Rep 22:388-396.

Bieri S, Potrykus I and Fütterer J. 2003. Effects of combined expression of antifungal barley seed proteins in transgenic wheat on powdery mildew infection. Mol Breed 11:37-48.

Blackshear PJ. 2002. Tristetraprolin and other CCCH tandem zinc-finger proteins in the regulation of mRNA turnover. Biochem Soc Trans 30:945-952.

Boman HG. 2003. Antibacterial peptides: basic facts and emerging concepts. J Intern Med 254:197-215.

Chakrabarti A, Ganapathi TR, Mukherjee PK and Bapat VA. 
2003. MSI-99, a magainin analogue, imparts enhanced disease resistance in transgenic tobacco and banana. Planta 216:587-596.

Chen C and Chen Z. 2002. Potentiation of developmentally regulated plant defense response by AtWRKY18, a pathogen-induced Arabidopsis transcription factor. Plant Physiol 129:706-716.

Conesa A, Götz S, García-Gómez JM, Terol J, Talón M and Robles M. 2005. Blast2GO: a universal tool for annotation, visualization and analysis in functional genomics research, Bioinformatics, 21(18):3674-3676.

De Smet K and Contreras R. 2005. Human antimicrobial peptides: defensins, cathelicidins and histatins. Biotechnol Lett 27:1337-1347.

Gudmundsson GH, Agerberth B, Odeberg J, Bergman T, Olsson B and Salcedo R. 1996. The human gene FALL39 and processing of the cathelin precursor to the antibacterial peptide LL-37 in granulocytes. Eur J Biochem 238:325332.

Gianinazzi-Pearson V, Dumas-Gaudot E, Gollotte A, TahiriAlaoui A and Gianinazzi S. 1996. Cellular and molecular defence-related root responses to invasion by arbuscular mycorrhizal fungi. New Phytol. 133:45-57.

Hammond-Kosack KE and Parker JE. 2003. Deciphering plant-pathogen communication: fresh perspectives for molecular resistance breeding. Curr Opin Biotech 14: 177-193.

Harrison MJ and Dixon RA. 1993. Isoflavonoid accumulation and expression of defense gene transcripts during the establishment of vesicular-arbuscular mycorrhizal associations in roots of Medicago truncatula. Mol. Plant-Microbe Interact. 6:643-654.

Jung YJ, Choi CS, Park JH, Kang HW, Choi JE, Nou IS, Lee SY and Kang KK. 2008. Overexpression of the pineapple fruit bromelain gene (BAA) in transgenic Chinese cabbage (Brassica rapa) results in enhanced resistance to bacterial soft rot. Electron J Biotechnol 11:1-8.

Jung YJ, Lee SY, Moon YS and Kang KK. 2012. Enhanced resistance to bacterial and fungal pathogens by overexpression of a human cathelicidin antimicrobial peptide (hCAP18/LL-37) in Chinese cabbage. Plant Biotechnol Rep. 6(1):39-46.

Kalde M, Barth M, Somssich IE and Lippok B. 2003. Members of the Arabidopsis WRKY group III transcription factors are part of different plant defence signaling pathways. Mol. Plant Microbe Interact. 16:295-305.

Kim LJ, Brian JJ, Antony B and Carolyn JS. 2003. The fasciclin-like arabinoglactan proteins of Arabidopsis. A multigene family of putative cell adhesion molecules. Plant Physiol. 133:1911-1925.

Kim YJ and Martin GB. 2004. Molecular mechanisms involved in bacterial speck disease resistance of tomato. Plant Pathol J. 20:7-12.

Kunkel BN. 2002. Brooks cross talk between signaling pathways in pathogen defense. Curr Opin Plant Biol 5:325-331.

Lee SC, Lim MH, Kim JA, Lee SI, Kim JS, Jin MN, Kwon SJ, Mun JH, Kim YK, Kim HU, Hur YK and Park BS. 2008. Transcriptome analysis in Brassica rapa under the abiotic stresses using Brassica $24 \mathrm{~K}$ oligo microarray. Mol. Cells 26:595-605.

Lee SC, Hwang IS, Choi HW and Hwang BK. 2008. Involvement of the pepper antimicrobial protein CaAMP1 gene in broad spectrum disease resistance. Plant Physiol. 143:1004-1020.

Liang H, Catranis CM, Maynard CE and Powell WA. 2002. Enhanced resistance to the poplar fungal pathogen, Septoria musiva, in hybrid poplar clones transformed with genes encoding antimicrobial peptides. Biotechnol Lett, 24:383-389.

Irizarry RA, Bolstad BM, Collin F, Cope LM, Hobbs B and Speed TP. 2003. Summaries of affymetrix GeneChip probe level data. Nucleic Acids Res. PN, e15.

Makandar R, Essig JS, Schapaugh MA, Trick HN and Shah J. 2006. Genetically engineered resistance to Fusarium head blight in wheat by expression of Arabidopsis NPR1. Mol Plant Microbe Interact 19:123-129.

Markus A, Belastegui-Macadam X and Kaldenhoff R. 2006. An attack of the plant parasite Cuscuta reflexa induces the expression of attAGP, an attachment protein of the host tomato. The Plant J. 48:548-556.

Park JM. 2005. The hypersensitive response: a cell death during disease resistance. Plant Pathol J. 21:99-101.

Prasad BD, Jha S and Chattoo BB. 2008. Transgenic indica rice expressing Mirabilis jalapa antimicrobial protein (Mj-AMP2) shows enhanced resistance to the rice blast fungus Magnaporthe oryzae. Plant Sci. 175: 364-371.

Reddy KV, Yedery RD and Aranha C. 2004. Antimicrobial peptides: premises and promises. Int J Antimicrob Ag. 24:536-547.

Rizhsky L, Liang H and Mittler R. 2002. The combined effect of drought and heat shock on gene expression in tobacco. Plant Physiol. 130:1143-1151.

Simmaco M, Mignogna G and Barra D. 1998. Antimicrobial 
peptides from amphibian skin: what do they tell us? Biopolymers 47:435-450.

Smith FD, Gadoury DM, Vaneck JM, Blowers A, Sanford JC, Van der Meij J and Eisenreich R. 1998. Enhanced resistance to powdery mildew in transgenic poinsettia conferred by antimicrobial peptides. Phytopathology, 88:S83 .

Sohn KH, Lee SC, Jung HW, Hong JK and Hwang BK. 2006. Expression and functional roles of the pepper pathogeninduced transcription factor RAV1 in bacterial disease resistance, and drought and salt stress tolerance. Plant Mol Biol 61:897-915.

Sorensen OE, Follin P, Johnsen AH, Calafat J, Tjabringa GS, Hiemstra PS and Borregaard N. 2001. Human cathelicidin, hCAP-18, is processed to the antimicrobial peptide LL37 by extracellular cleavage with proteinase 3 . Blood, 97:3951-3959.

Travis SM, Anderson NN, Forsyth WR, Espiritu C, Conway BD, Greenberg EP, McCray PB, Lehrer RI, Welsh MJ and Tack BF. 2000. Bactericidal activity of mammalian cathelicidin-derived peptides. Infect Immun, 68:2748-2755. Yamasaki K, Schauber J, Coda A, Lin H, Dorschner RA, Schechter NM, Bonnart C, Descargues P, Hovnanian A and Gallo RL. 2006. Kallikrein-mediated proteolysis regulates the antimicrobial effects of cathelicidins in skin. Fed Am Soc Exp Biol J. 20:2068-2080.

Wang L, Xu Y, Zhang C, Ma Q and Joo S-H. 2008. OsLIC, a novel CCCH-Type zinc finger protein with transcription activation, mediates rice architecture via brassinosteroids signaling. PLoS ONE 3(10):e3521.

Workman C, Jensen LJ, Jarmer H, Berka R, Gautier L, Nielser HB, Saxild HH, Nielsen C, Brunak S and Knudsen S. 2002. A new non-linear normalization method for reducing variability in DNA microarray experiments. Genome Biol. P, research, 0048.1-0048.16.

Zhang X, Dai Y, Xiong Y, DeFraia C, Li J, Dong X and Mou Z. 2007. Overexpression of arabidopsis MAP kinase kinase 7 leads to activation of plant basal and systemic acquired resistance. Plant J 52:1066-1079. 